\title{
A Novel Approach to Face Detection using Image Parsing and Morphological Analysis
}

\author{
Abhishek Maity $^{\# 1}$, Sayan Dasgupta ${ }^{\# 2}$, Debjit Paul ${ }^{\# 3}$ \\ ${ }^{\#}$ Department of Computer Science \& Engineering \\ Guru Nanak Institute of Technology, Kolkata, India
}

\begin{abstract}
The main objective of the paper is to propose an algorithm on extraction of some fundamental information of an image efficiently and then finally use that to detect the human face on the image. The method proposed is based on assumption that the image contains the frontal face.The described method widely-used edge-based features. Firstly, the skin region is detected using a colour based algorithm and Brightness preserving histogram equalization techniques operated on RGB to determine the presence of human and the head region is calculated.Lastly, probable calculated head region is extracted using Sobel Edge-Detection and matched with our trained database files for further classification of the segmented image
\end{abstract}

Keywords - Content-based image retrieval (CBIR), Brightness preserving fuzzy histogram equalization, Image segmentation, Image cropping.

\section{INTRODUCTION}

The most searched item found online is of human being. And sensing the occurrence of human has always been an intricate and complex job. In recent times, many image processing applications have utilized databases of images to support image manipulation and synthesis operation. [1] [2] [3]. However, humans face have been proven to be a much more difficult object to detect because of wide variability in appearance due to articulation and illumination conditions that are common in outdoor scenes.

In our paper, we have used skin-colour based algorithm to determine the human face in the image. As colour processing is much faster than other facial features we have decided to with human skin colour uncovering (RGB colour-space) as a first step of achieving the goal. Tracking human faces using colour has quite a few problems like the colour illustration of face gained through a camera is subjective to many factors (ambient light, object movement, etc.), different cameras produce considerably varies colour values even for the same person under the same lighting conditions and skin colour differs from person to person. In order to use colour as a feature for face tracking, we solved these problems using Brightness preserving dynamic fuzzy histogram equalization [4].To make it more accurate, we have used edge based human detection algorithm to find number of white pixels present in the image.

\section{RELATED WORK}

Human detection is the most challenging task in image processing. There has been a lot of work done concerning the recognition of human. The skin colour recognition plays a vital part in the human detection especially in face detection. This property is very useful in the cases of detecting the skin region or non- skin region in an image. There are various colour spaces such as RGB (Red Green Blue), NTSC, YCbCr, HSV, CMY, HIS, YUV, CIE-Lab etc. The skin colour for example for fair complexion is determined with some rules [5] [6] which describe the skin cluster in the RGB colour space. But this method detects every possible region that has the predescribed RGB values even if it's not the face of human. Apart from that there are many databases present online but building an image database is still a non-trivial task because very few of these images are annotated or perfect for making the database. Recently, algorithms have been proposed to automatically analyse online images to build large scale databases for skies [3] and faces [2]. However building and finding such a database is still very rare to have. Detecting human through edge-detection has been done before. Depending upon the shape of human and being subjective to the statistically found data over the edges of an image, human is detected.[7] On top of that another CBIR based work has been proposed to uniquely identify human from an image[8]. Here [8] L*a*b* colour space has been used and pixel-count was the key factor to determine the presence of human. But this was highly dependent upon image filtering technique. And an approximation was used in every case where multiple human were present making the system less-reliable.

\section{HUMAN SKIN COLOUR DETECTION}

With the help of heuristic rules, it is determined, whether a certain pixel of input image corresponds to the skin colour. Note that the original face detection algorithm was developed to work best under standard daylight illumination (CIE illumination D65).As stated in [14] [16], we adopted the same procedure with minimal changes. We have introduced Brightness Preserving Dynamic Fuzzy Histogram Equalization [4] for better efficiency. After the skin colour classification is done for every pixel of the image, the skin region segmentation takes place (Figure 1).Unsuitable regions are then eliminated on the basis of colour distribution over the face region. Remaining regions represents face. The skin 
colour for example for fair complexion is determined with the following rules which describe the skin colour in the RGB colour-space.

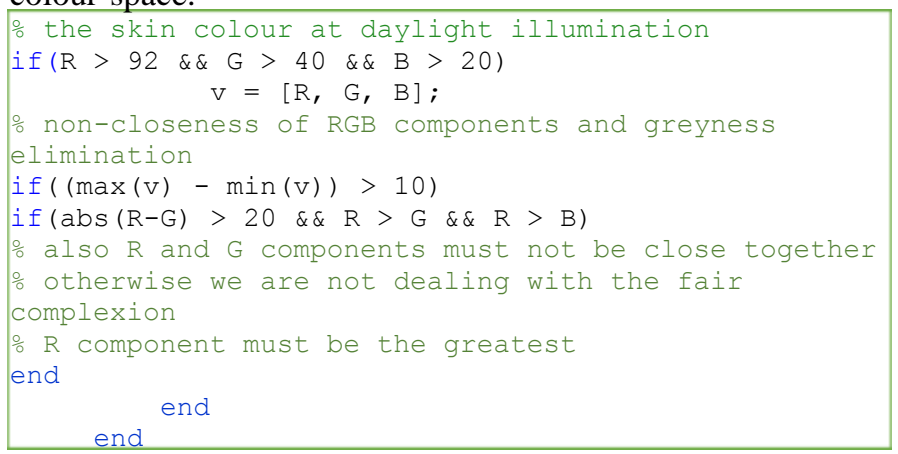

MATLAB code snippet of the proposed logic

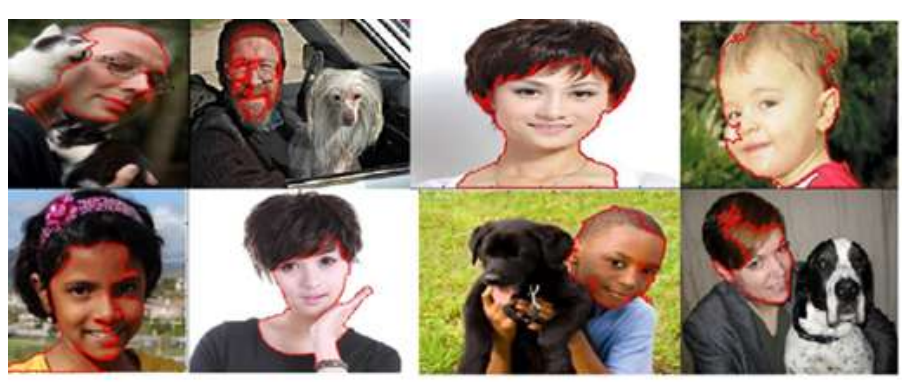

Figure 1 from top-left $\boldsymbol{a} \boldsymbol{b} \boldsymbol{c} \boldsymbol{d}$ bottom-left $\boldsymbol{e} \boldsymbol{f} \boldsymbol{g} \boldsymbol{h}$

This is how we get the basic information for face candidates. But this approach detects every possible skinregion within the image (See fig. 2), and this detection is erroneous as even non-human image are also calculated. In fig (2.a) the detected part is a dog. In other case human-arms (2.b), floor (2.b)is also sensed as human skin region. Now, to detect only human faces it was necessary to individually analyse each tracked sub-image [possible skin- regions covered by isolated red boundaries] for being a human facial area. To do this, we cropped every detected sub-part of the image for additional analysis as depicted further.

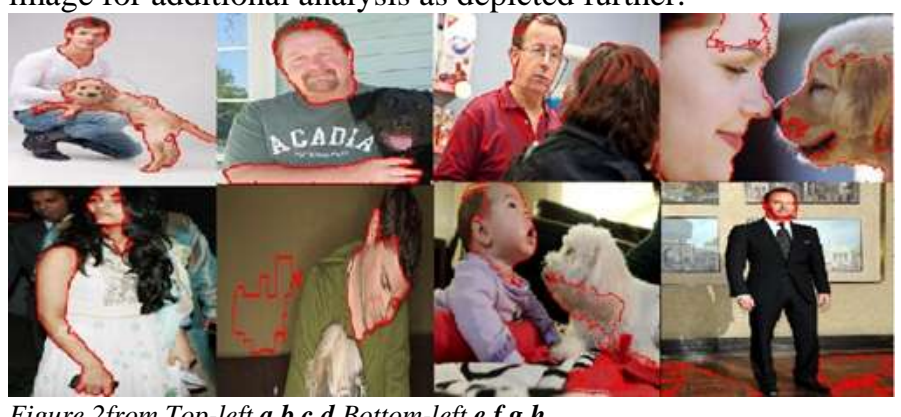

IV.DATA ANALYSIS AND PRE-PROCESSING

It has been seen that, in general, the ratio of an operated image size and the number of white pixels (after edgedetection) present in that image stays within a finite range. So, we downloaded and congregated as many as human-face possible and then do an edge-based Sobel detection to calculate the number of white pixels present in the image. After detecting and calculating it, we compute the ratio of the image size and the number of white pixels present in the picture. Depending on the statistics that we have found, we made the database having the image size and corresponding white pixels as prime characteristics.

Now data obtained from each target image is matched with the database for nearest value i.e. depending upon the size of the target image or user-given image (after cropping according to skin detection), nearest image-size in the database is searched and then number of white pixel present in user given image is checked with our database. The picture is marked as human only if the difference in the number of white pixel present in the user-given image and in the database is within a very nominal threshold range. The threshold value is decided to be 30 , making the system much efficient.

\section{Human Skin COlour Detection}

After various necessary and essential morphological operations has been applied. At first hole filling operations (figure 4) have been applied to fill any gaps [9] in the individual blobs. In case for narrowly connected blobsopening, the image obtained bwperim function [15] is subtracted from the main binary image for a two to three times; thus finally the narrowly connected regions are separated. As the subtraction leads to loss in size of the individual blobs the final morphological operation includes a dilate type function [10] to maintain a recognizable size of the individual skin detected blobs.

Few additional measures are also applied to determine the probability of a skin region being a face region [11] [12] [13].Two region properties -eccentricity and box ratio are used to classify and examine the shape of each skin area.

The box ratio [10] property is defined simply as the ratio width to height of the bounding box area. Through trial and error on the train setstheoutcome that is lying between 1.1 and 0.4 is obtained. Values above 1.1 would not suggest a face since human faces are vertically oriented with a longer length than width. In the meantime, values below 0.4 misclassify arms, legs or other elongated objects as faces. The eccentricity property measures the minor to major axis ratio of the bounding elliptical region. Eccentricity between 0.25 and 0.97 are considered to be of better range for face areaclassification. This property works in a way as box ratio.Though,it is more sensitive to the shape and takes various poses and rotations of face into account. At last the probable face region (figure 5) is detected.

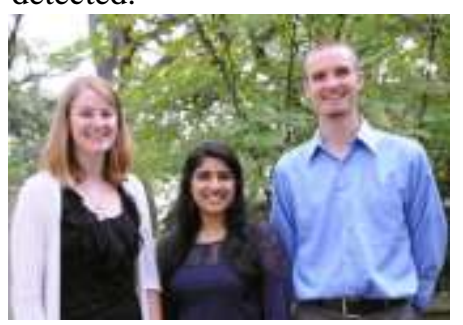

Figure 3 sample image 


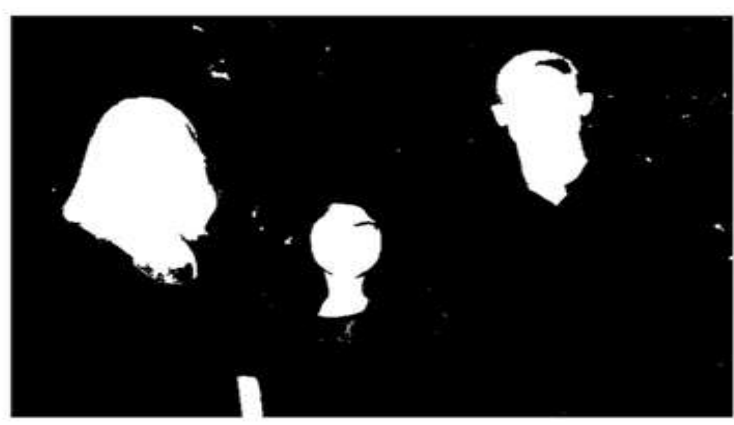

Figure 4

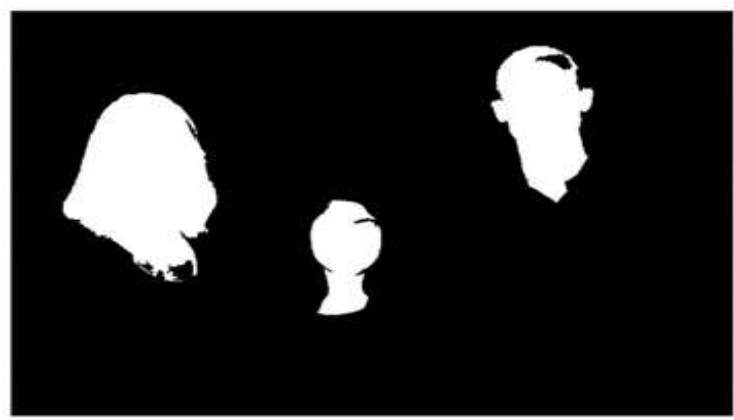

Figure 4

VI. FACE REGION CROPPING

After getting the face region first, the conversion of the original intensity image $(I(x, y))$ to gray scale image $\lim (x, y))$.then the Sobel edge detector is applied to the gray scale image $\operatorname{im}(x, y), O \leq x \leq M-1$ and $O \leq y \leq N-$ 1 .Let $K(x, y)$ denote the obtained edge image where $K(x, y)=1$ if $(x, y)$ is an edge pixel and otherwise $K(x, y)=0$. Next, the number of 1 of each column $\mathrm{x}$ and each row y are counted, and stored these as $V(x)$ and $H(y)$.Now the $V(x)$ and $H(y)$ are computed $\mathrm{b}$.

$$
\begin{array}{rr}
V(x)=\sum_{\substack{y=0 \\
M-1}}^{N-1}(x, y) & \text { for vertical direction } \\
H(y) & =\sum_{x=0}^{M(x, y)} \quad \text { for horizontal direction }
\end{array}
$$

Now the next step is to find out the left and right boundary of head are given by the smallest and largest values of $\mathrm{x}$ such that $V(x) \geq V(x 0) / 3$ where $\mathrm{x} 0$ denotes the column $\mathrm{x}$ with the largest $V(x)$.And, the y-position ymin of the upper boundary of the head is given by the smallest y such that $H(y) \geq 0.05 *(x R-x L)$.Finally, we find out the $y$-position $y \max$. Then ymax computed by

$$
y \max =\max K(x, y)
$$

If the above procedure is applied to extract the face region from the image of figure 6 then figure $7 a, 7 b$ and $7 c$ will be the output images.

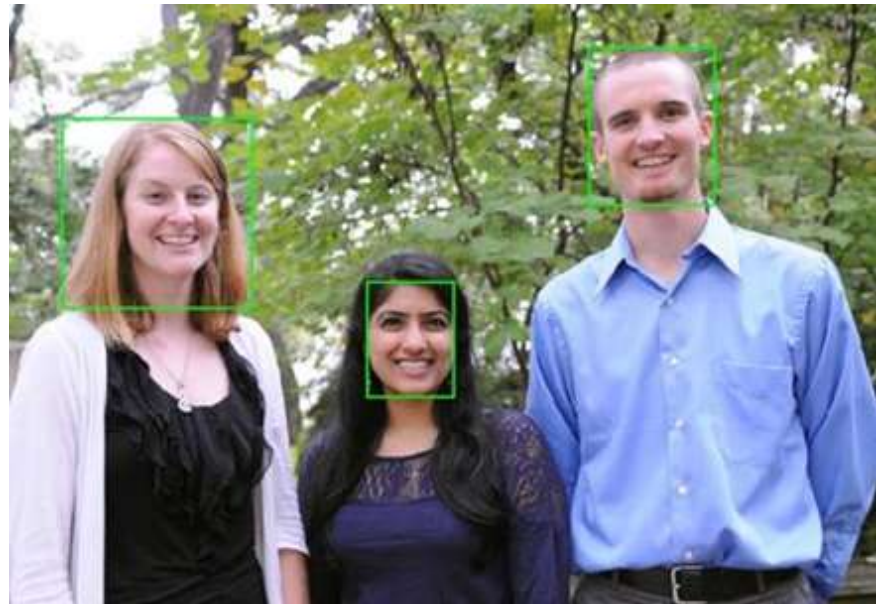

Figure 6 image after operation in MATLAB
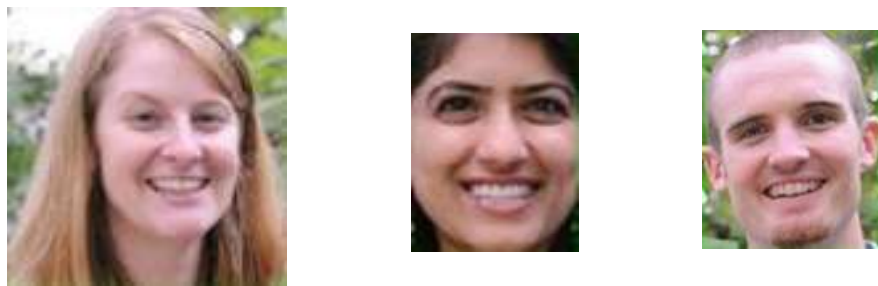

Figure 7 from left-to-right $\mathbf{a}, \mathbf{b}, \mathbf{c}$ resultant cropped images

VII. PROPOSED ALGORITHM

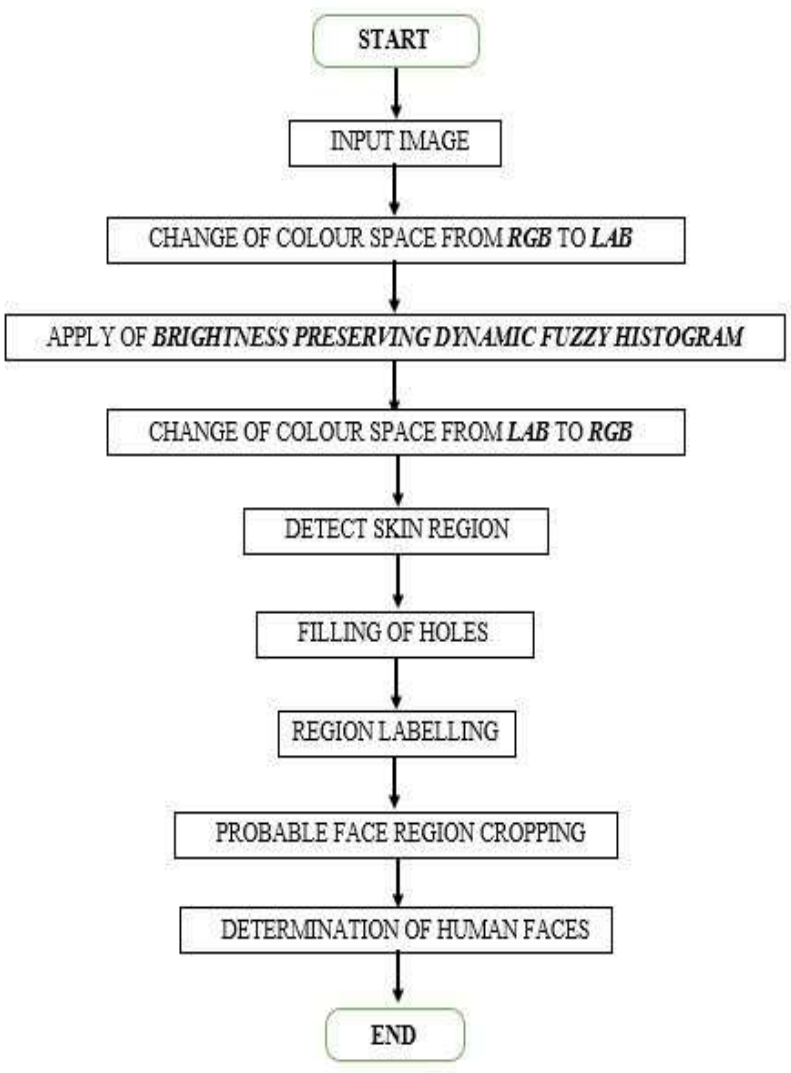


VIII. EXPERIMENTAL RESULTS

The model based on the proposed algorithm was evaluated on a face detection system using a test data set of 20 images. To evaluate the experiment, we used the method introduced by Thakur et al. [12]. Two performance metrics are defined to gauge the success of the schemes. False Detection Count (FDC) is defined as the number of false detections over the total number of detections.

$$
F D C=\frac{\text { number of false detections }}{\text { total number of detections }} \times 100
$$

Detection Success Count (DSC) is defined as the number of correctly detected faces over the actual number of faces in the image.

$$
D S C=\frac{\text { number of correctly detected faces }}{\text { total number of faces }} \times 100
$$

Where the number of correctly detected faces are equivalent to the number of faces minus the number of false dismissals.

\section{A. Test Images}
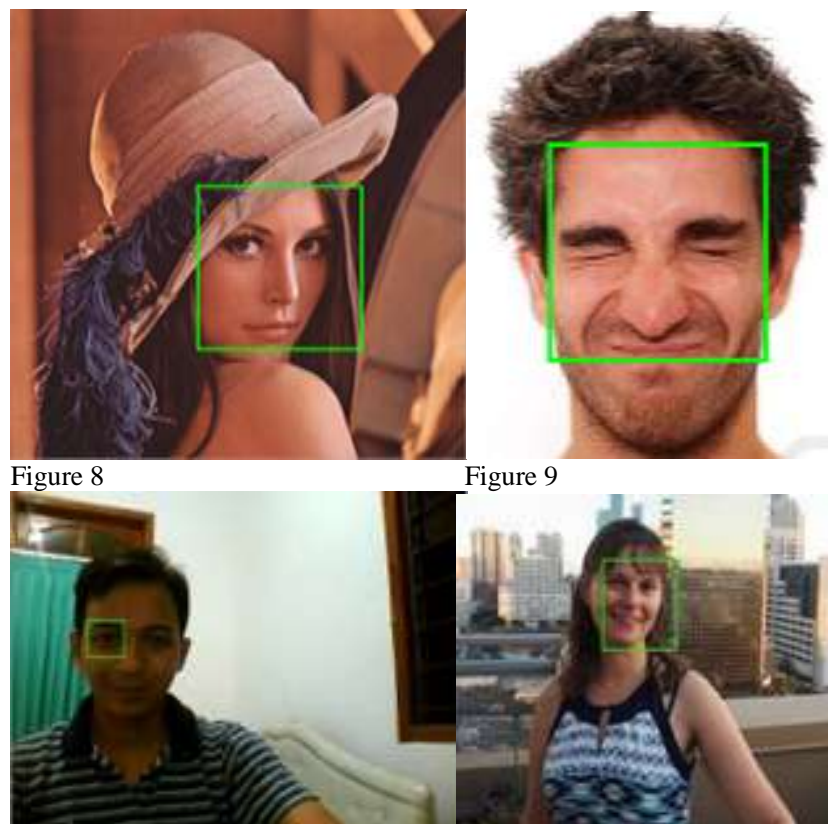

Figure 9

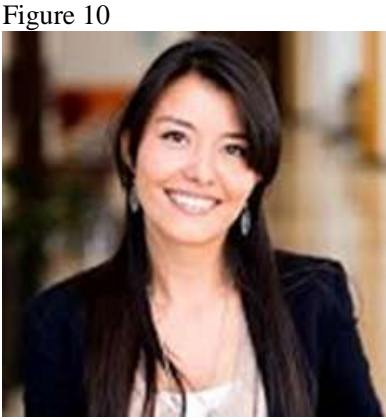

Figure 12

Figure 13

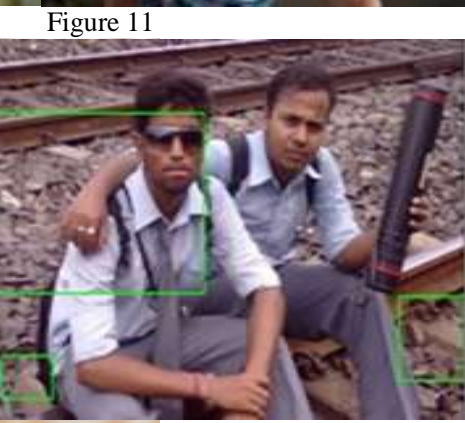

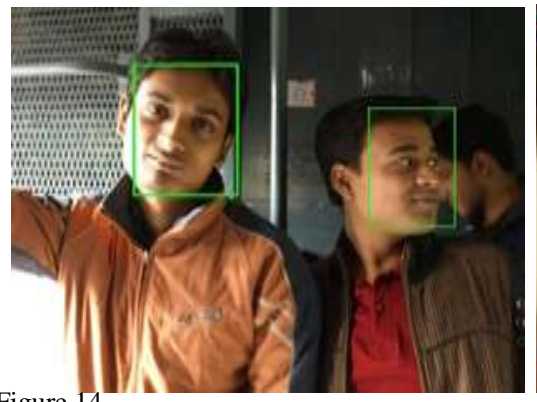
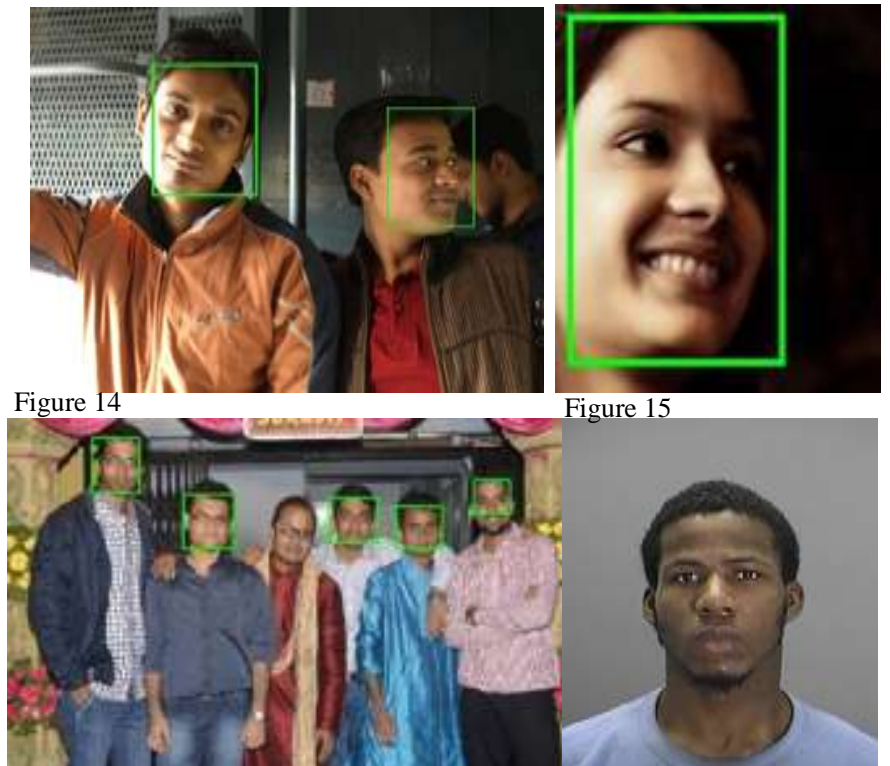

Figure 15
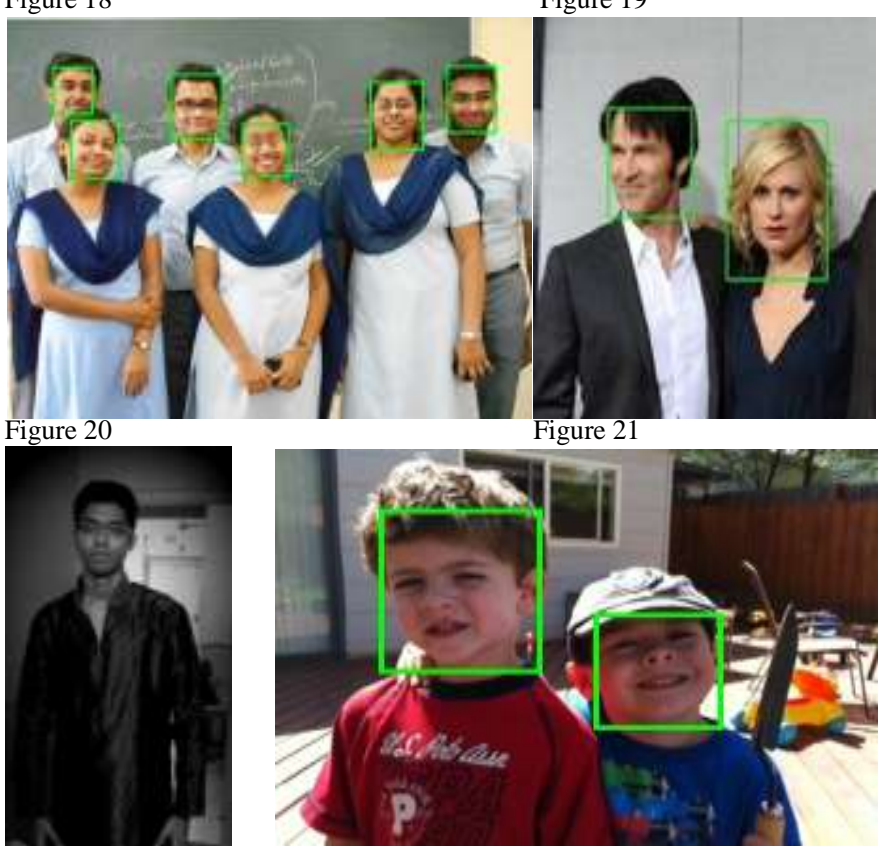

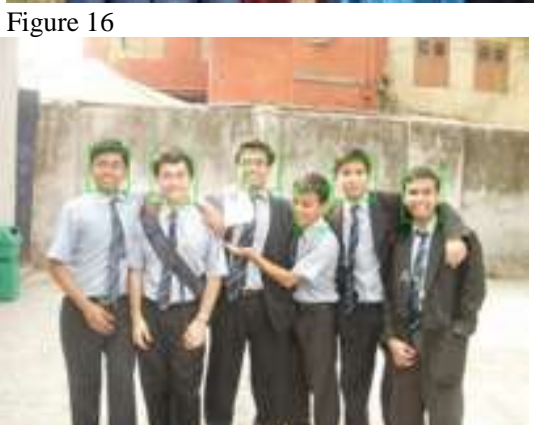

Figure 18

Figure 23

Figure 17

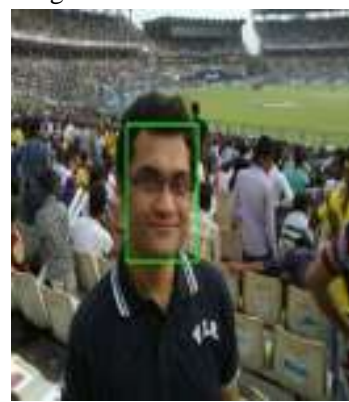

Figure 19
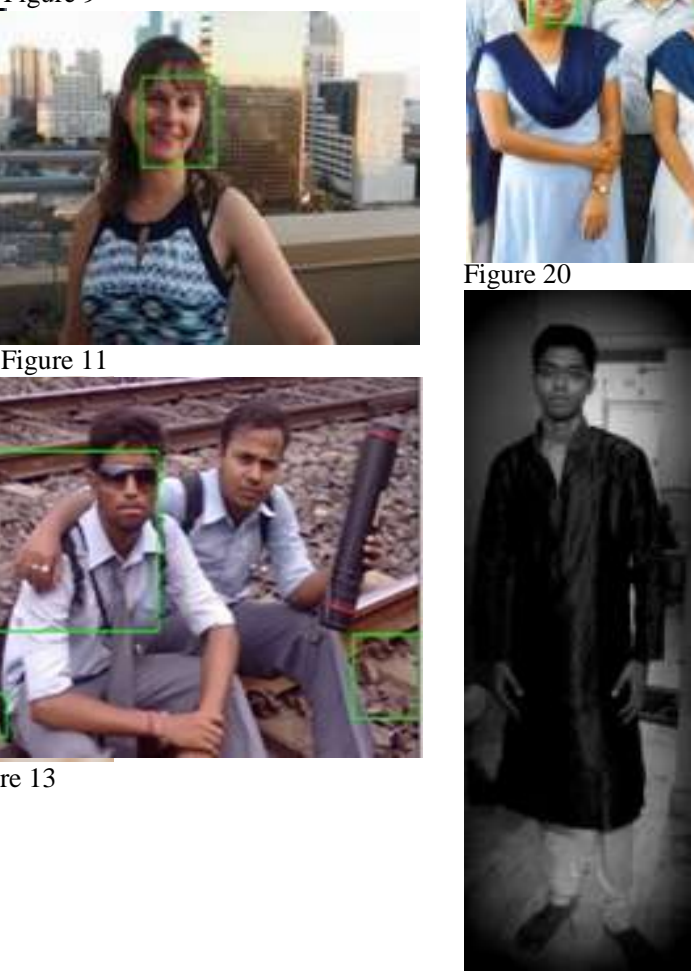

Figure 11

Figure 22 
International Journal of Computer Trends and Technology (IJCTT) - volume 23 Number 4-May 2015
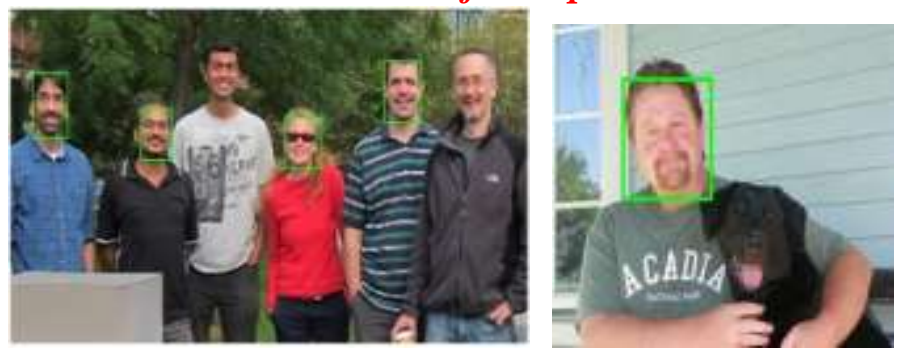

Figure 24

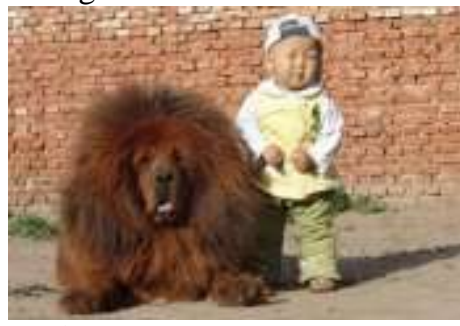

Figure 26

B. System Accuracy

TABLE I. Performance of Face Region Marking

\begin{tabular}{|c|c|c|}
\hline Figure & FDC (\%) & DSC (\%) \\
\hline 8 & 0 & 100 \\
\hline 9 & 0 & 100 \\
\hline 10 & 100 & 0 \\
\hline 11 & 0 & 100 \\
\hline 12 & 0 & 0 \\
\hline 13 & 66.66 & 50 \\
\hline 14 & 0 & 66.66 \\
\hline 15 & 0 & 100 \\
\hline 16 & 0 & 83.33 \\
\hline 17 & 0 & 0 \\
\hline 18 & 0 & 100 \\
\hline 19 & 0 & 100 \\
\hline 20 & 0 & 100 \\
\hline 21 & 0 & 100 \\
\hline 22 & 0 & 0 \\
\hline 23 & 0 & 100 \\
\hline 24 & 0 & 66.66 \\
\hline 25 & 0 & 100 \\
\hline 26 & 0 & 0 \\
\hline 27 & 0 & 100 \\
\hline & & \\
\hline
\end{tabular}

Therefore, Overall Performance $(\mathrm{OP})=\{$ Total success rate $/$

Total number of images $\}=(1533.31 / 20)=76.66 \%$

\section{CONCLUSIONS}

Though there are many modern and accurate software today for facial recognition but this approach is totally new and also less complex and simplest to handle. In our approach, we have used the techniques of Content Based Image Retrieval (CBIR).The content here is the blend of colours, shapes, textures, or any other information that can be derived from the image itself. Besides the images submitted by the user could either be cropped as desired by the user or the entire image can be used for information retrieval. Some of the images are manually changed to get a comparative difference. And even the manually changed images show the perfect result when gone through the series of operations that has been stated above. It has to be noted that our proposed algorithm is gesture independent i.e. it does not depend upon the variations of facial expression but the face itself. Apart from that this algorithm detects multiple human efficiently. So, if anyone provides any non-human (strictly speaking nonliving) objects then the image remains undetected thus conveying the idea that there was only no-human objects are present. So our project of identifying human, in turn, can detect if non-human objects are present in an image or not

\section{ACKNOWLEDGMENT}

The authors are grateful to Utsav Dawn and Ayan Kumar Saha from Department of Computer Science \& Engineering, Guru Nanak Institute of Technology, Kolkata, India, for helping out generously on trained data-sets. The authors would like to thank the anonymous reviewers' toward improvement of the paper.

\section{REFERENCES}

[1] James Hays and Alexei A. Efros. 2007. Scene completion using millions of photographs. ACM Trans. Graph. 26, 3, Article 4 (July 2007). DOI=10.1145/1276377.1276382

[2] Dmitri Bitouk, Neeraj Kumar, Samreen Dhillon, Peter Belhumeur, and Shree K. Nayar. 2008. Face swapping: automatically replacing faces in photographs. ACM Trans. Graph. 27, 3, Article 39 (August 2008), 8 pages.DOI=10.1145/1360612.1360638

[3] Litian Tao, Lu Yuan, and Jian Sun. 2009. SkyFinder: attribute-based sky image search. ACM Trans. Graph. 28, 3, Article 68 (July 2009), 5 pages. DOI=10.1145/1531326.1531374

[4] Sheet, D.; Garud, H.; Suveer, A.; Mahadevappa, M.; Chatterjee, J., "Brightness preserving dynamic fuzzy histogram equalization," Consumer Electronics, IEEE Transactions on , vol.56, no.4, pp.2475,2480, November 2010 doi: 10.1109/TCE.2010.5681130

[5] P. Peer, F. Solina, "An Automatic Human Face Detection Method," in Proceedings of Computer Vision Winter Workshop, pp. 122-130, Rastenfeld, Austria, 1999.

[6] Solina, F.; Peer, P.; Batagelj, B.; Juvan, S., "15 seconds of fame - an interactive, computer-vision based art installation," Control, Automation, Robotics and Vision, 2002. ICARCV 2002. 7th International Conference on , Control, Automation, Robotics and Vision vol.1, no., pp.198,204 vol.1, 2-5 Dec. 2002 doi 10.1109/ICARCV.2002.1234820

[7] Moon, H.; Chellappa, R.; Rosenfeld, Azriel, "Optimal edge-based shape detection," Image Processing, IEEE Transactions on , vol.11, no.11, pp.1209,1227, Nov 2002 doi: 10.1109/TIP.2002.800896

[8] Mukherjee, S.; Chatterjee, A.; Dhar, M., "Image parsing: An advanced approach for image identification," Computing and Communication Systems (NCCCS), 2012 National Conference on , vol., no., pp.1,6, 2122 Nov. 2012 doi: 10.1109/NCCCS.2012.6413022

[9] Lakshmi, HC Vijay, and S. Patil Kulakarni. "Face Localization and Detection Algorithm for Colour Images Using Wavelet Approximations." Computer Vision and Information Technology: Advances and Applications (2010): 305 
International Journal of Computer Trends and Technology (IJCTT) - volume 23 Number 4-May 2015

[10] Gonzalez, Rafael C., Richard Eugene Woods, and Steven L. Eddins. Digital image processing using MATLAB. Pearson Education India, 2004

[11] Farkas, Leslie G. Anthropometry of the Head and Face. New York, NY: Raven, 1994. Print.

[12] Thakur, S.; Paul, S.; Mondal, A.; Das, S.; Abraham, A., "Face detection using skin tone segmentation," Information and Communication Technologies (WICT), 2011 World Congress on , vol., no., pp.53,60, 11-14 Dec. 2011 doi: 10.1109/WICT.2011.6141217

[13] Ruangyam, P.; Covavisaruch, N., "An efficient region-based skin color model for reliable face localization," Image and Vision Computing New Zealand, 2009. IVCNZ '09. 24th International Conference, vol., no., pp.260, 265, 23-25 Nov. 2009 doi: 10.1109/IVCNZ.2009.5378401

[14] Kovac, J.; Peer, P.; Solina, F., "Human skin color clustering for face detection," EUROCON 2003. Computer as a Tool. The IEEE Region 8, vol.2, no., pp.144, 148 vol.2, 22-24 Sept. 2003 doi: 10.1109/EURCON.2003.1248169

[15] Find perimeter of objects in binary image, MATLAB bwperim, MathWorks India. Retrieved May 25, 2015 from http://in.mathworks.com/help/images/ref/bwperim.html

[16] Kovac, J.; Peer, P.; Solina, F., "Illumination independent color-based face detection," Image and Signal Processing and Analysis, 2003. ISPA 2003. Proceedings of the 3rd International Symposium on , vol.1, no., pp.510,515 Vol.1, 18-20 Sept. 2003 doi: 10.1109/ISPA.2003.1296950 